\title{
Changement institutionnel et agriculture à Mayotte
}

Les impacts d'une intégration renforcée à la République française sur l'activité agricole des ménages

Institutional change and agriculture in Mayotte. The Impacts of its integration into the french republic on agricultural activity.

Jean-Michel Sourisseau, Philippe Bonnal et Perrine Burnod

\section{(2) OpenEdition}

Journals

Édition électronique

URL : http://journals.openedition.org/economierurale/525

DOI : 10.4000/economierurale.525

ISSN : 2105-2581

Éditeur

Société Française d'Économie Rurale (SFER)

Édition imprimée

Date de publication : 1 juin 2008

Pagination : 60-74

ISSN : 0013-0559

\section{Référence électronique}

Jean-Michel Sourisseau, Philippe Bonnal et Perrine Burnod, « Changement institutionnel et agriculture à Mayotte », Économie rurale [En ligne], 303-304-305 | Janvier-juin 2008, mis en ligne le 30 juin 2010, consulté le 19 avril 2019. URL : http://journals.openedition.org/economierurale/525; DOI : 10.4000/ economierurale.525 


\title{
Changement institutionnel et agriculture à Mayotte Les impacts d'une intégration renforcée à la République française sur l'activité agricole des ménages
}

\author{
Jean-Michel SOURISSEAU et Philippe BONNAL • CIRAD ES, Environnements et Sociétés, \\ Unité propre de recherche Action collective, politiques et marchés, Arena
}

Perrine BURNOD • CIRAD ES, Environnements et Sociétés, Unité propre de recherche Action collective, politiques et marchés, Arena, Marchés, Organisations, Institutions et Stratégies d'Acteurs (MOÏSA), Montpellier

$L_{\text {in }}^{\text {it }}$ 'île de Mayotte connaît une transition institutionnelle d'envergure. Cette ancienne colonie française de l'océan Indien, après avoir refusé l'indépendance au sein de l'ensemble comorien, a réaffirmé son choix en 2001 pour une intégration totale dans la République française ${ }^{1}$. Elle est donc engagée dans un processus volontaire d'alignement de son cadre légal sur celui de la France. Ce changement institutionnel concerne en premier lieu les institutions ${ }^{2}$ dites formelles ${ }^{3}$ et peut être qualifié d'exogène dans le sens où il ne résulte ni du jeu progressif d'organisations locales sur les décideurs politiques, ni d'une formalisation ou d'une transformation d'institutions préexistantes localement.

Le processus de transition de Mayotte est atypique ; il se distingue fondamentalement de celui des pays dits « du Sud » où des États affaiblis doivent mettre en place des réformes macro-économiques en grande

1. Ce processus pourrait la conduire au statut de département à l'horizon 2010.

2. Une institution peut être définie comme un ensemble de relations ordonnées entre les individus qui définissent leurs droits, leurs expositions aux droits des autres, leurs privilèges et leurs responsabilités (Schmid, 2004).

3. North (1990) distingue les institutions formelles constituées par les règles politiques, juridiques et économiques officielles (lois) des institutions informelles conformées par les codes, normes de conduite et aux conventions, qui ont une forte dimension culturelle et sociale.

partie imposées par des institutions financières internationales ou encore de celui des ex-pays à économie planifiée qui privatisent des pans entiers de leurs économies. L'île vit au contraire un renforcement de l'action de l'État, qui vise à rapprocher la société mahoraise et le fonctionnement de son économie des modèles métropolitains. Ce processus met en évidence les oppositions entre les institutions locales africaines et musulmanes à la base de l'organisation sociale locale et les institutions républicaines. L'instauration ou le renforcement des institutions formelles sont en effet synonymes d'une redéfinition des droits des individus, impliquant à la fois de nouvelles contraintes mais aussi de nouvelles opportunités (Schmid, op. cit.). Au-delà d'une mise aux normes vers les standards métropolitains, cette dynamique de rattrapage institutionnel a engendré d'un point de vue général l'augmentation de l'offre d'emploi et des niveaux de salaire, l'amélioration des services publics, la mise en place d'aides sociales et publiques plus conséquentes (allocations, bourses, subventions), se traduisant pour les ménages par une amélioration significative de tous les indicateurs de développement économique et social.

Ces changements institutionnels concernent directement l'activité agricole. Accompagné par les souhaits d'une partie des cadres politiques et des services techniques étatiques, l'alignement sur l'organisation de la métropole signifie l'accès 
aux subventions agricoles, la régularisation du foncier, la rémunération des travailleurs agricoles selon le taux légal et le respect des normes sanitaires. Le but clairement affiché, notamment dans une situation de croissance démographique et de monétarisation de l'économie, est de faire évoluer l'agriculture vers des formes plus productives et rémunératrices, plus conformes au modèle métropolitain. Sur la base d'un référentiel d'analyse institutionnelle, l'objectif de cet article est d'identifier les impacts de ce changement institutionnel sur la gestion de l'agriculture au niveau des ménages et de les discuter.

Après avoir rappelé la nature des changements à l'œuvre, nous examinerons les différentes fonctions assignées par les ménages ruraux à l'activité agricole en soulignant leur relative permanence. Nous étudierons ensuite les adaptations de l'organisation de l'activité agricole en nous centrant sur les recompositions de la gestion du foncier et du travail agricole, ainsi que sur les principes de valorisation de la production. Enfin, nous discuterons les raisons expliquant le comportement des ménages face au changement institutionnel.

\section{Les conséquences attendues de l'intégration dans la République française}

Mayotte est une île de $374 \mathrm{~km}^{2}$ située dans l'archipel des Comores et comptant 175000

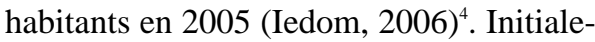
ment constituée de Bantous originaires d'Afrique de l'Est, sa population se métisse à la suite de différentes vagues migratoires, dont certaines en provenance du MoyenOrient qui vont profondément marquer l'organisation sociale par leurs apports arabomusulmans. La société mahoraise reçoit aussi des influences européennes dès le $\mathrm{XV}^{\mathrm{e}}$ siècle à travers des contacts avec des

4. Iedom : Institut d'émission des départements d'outre-mer. explorateurs et marchands, et surtout à partir du début de la colonisation française en 1841. La domination française se traduit néanmoins par la faiblesse de l'immigration et des investissements métropolitains, avec pour corollaire un impact limité sur les rapports sociaux de production préexistants. Marqués par la prédominance d'une économie agricole d'autosubsistance, ceuxci restent schématiquement fondés sur l'appartenance à une communauté villageoise et à un lignage, au sein desquels se répartit le foncier et se gère l'utilisation des biens agricoles. L'Islam, très prégnant, complète le cadre de référence des droits et obligations réglant l'organisation sociale.

En décembre 1974, lors du référendum d'indépendance des Comores, les Mahorais votent à une courte majorité le maintien de l'île dans le territoire français. Mayotte réaffirmera ensuite à plusieurs reprises par les urnes son attachement à la France et sa volonté d'une séparation définitive et irrévocable avec la jeune République Islamique des Comores. Mais malgré ces signes forts et renouvelés, l'île est maintenue pendant 25 ans dans une situation statutaire ambigüe par rapport à la France. Ce n'est qu'après maints atermoiements et de longs arbitrages quant au rythme des réformes à mettre en place que le processus de départementalisation, lancé en 2001, consacre son intégration définitive dans la métropole. Ce tournant s'accompagne d'un renforcement des politiques publiques visant à rapprocher la société mahoraise et le fonctionnement de son économie des modèles métropolitains, mettant ainsi en évidence les oppositions entre les règles africaines et arabo-musulmanes de la gestion coutumière et les règles républicaines. Si le mouvement est global, il touche particulièrement le secteur agricole.

Le passage du droit local musulman au droit commun se traduit ainsi par l'engagement d'une régularisation foncière visant à substituer la propriété individuelle de droit romain à la gestion collective coutumière et religieuse. Le rattrapage volontariste des 
salaires s'accompagne d'une hausse significative des revenus des ménages et, par suite, d'une modification de leurs habitudes de consommation. L'introduction du système métropolitain de répartition avec de nouveaux impôts et taxes mais aussi de nouvelles aides sociales (allocations familiales, logement, chômage, etc.) perturbe les logiques communautaires de gestion de la solidarité qui passent en partie par les échanges de produits agricoles. L'ajustement de la législation locale sur le travail remet en cause certains arrangements, notamment entre propriétaires ${ }^{5}$ et ouvriers agricoles. Ajoutons enfin que les niveaux de salaires et de transferts publics attirent à Mayotte un flux non maîtrisé de migrants clandestins, principalement Anjouanais, qui détermine pour beaucoup les conditions et la dynamique du marché du travail ${ }^{6}$.

La mise aux normes institutionnelle se traduit pour les ménages agricoles mahorais par une série de ruptures, mais elle garantit aussi une augmentation de leur revenu et leur ouvre un champ d'opportunités a priori très attractives : accès à l'éducation et à l'emploi salarié dans le secteur public ou parapublic, création d'activités dans le secteur des services, promotion de l'entreprenariat, etc. Dans ce contexte de transition institutionnelle et de forte pression démographique ${ }^{7}$, des changements structurels étaient attendus, parmi lesquels la concentration de l'activité agricole avec l'émer-

5. La « propriété » recouvre ici, par extension, la détention d'un droit d'usage dans le droit musulman et coutumier et la propriété de droit commun, matérialisée par la détention d'un titre foncier.

6. Les clandestins représentent aujourd'hui un tiers de la population de l'île. Les réponses de l'État français et de l'exécutif local aux tensions liées à l'immigration consistent principalement à améliorer la surveillance des frontières et à intensifier la répression ; plus de 10000 clandestins sont reconduits aux Comores chaque année.

7. La population de Mayotte a quadruplé en trente ans. Le taux de croissance annuel moyen est de $3,6 \%$ et la densité de population atteint 470 habitants $/ \mathrm{km}^{2}$. gence d'exploitations strictement marchandes et le recul massif d'une activité agricole principalement destinée à l'autoconsommation et aux dons.

\section{La relative permanence des fonctions assignées à l'activité agricole}

Si le déclin des productions de rentes traditionnelles (vanille et ylang-ylang ${ }^{8}$ ) et le délaissement de la cocoteraie ${ }^{9}$ du fait de la chute des prix du coprah sont significatifs, on note effectivement le développement de parcelles de maraîchage ${ }^{10}$, de bananiers ou de fruitiers en monoculture tournées vers le marché. Mais ce développement concerne une minorité de ménages et demeure limité. La faiblesse des stratégies de commercialisation est patente et les résultats des dispositifs d'encouragement à la modernisation des structures agricoles mis en place depuis les années 1980 sont mitigés. Ainsi les groupements de producteurs créés à l'initiative de l'administration pour commercialiser les produits ont périclité (Losch et Sourisseau, 2002). De même, seul un tiers des ménages agricoles déclare vendre ou échanger une partie de leur production végétale et plus de $60 \%$ d'entre eux ne le font que s'ils ont des surplus saisonniers (Huet, 2004) ; seuls $6 \%$ déclarent vendre des produits issus de l'élevage ou des animaux. Finalement, d'un point de vue macroéconomique, l'agriculture est estimée contribuer à $9 \%$ du PIB mahorais en 2001 (Frouté et Massuyau, 2005) $)^{11}$ et est jugée en net recul, voire menacée.

8. Arbre cultivé pour ses fleurs utilisées en parfumerie.

9. Celle-ci occupe encore 3400 ha, mais les arbres sont âgés et peu productifs (Huet, 2004).

10. Les surfaces maraîchères sont évaluées à 40 ha en 2003 très souvent à l'initiative de producteurs en situation irrégulière.

11. Frouté O., Massuyau B. (2005). Évaluation du PIB à Mayotte. Document de travail Cédérom n ${ }^{\circ} 1$, Insee, Iedom, AFD. Mamoudzou, 23 p. 
Pourtant, les études disponibles mettent en évidence le maintien, voire le développement de l'activité agricole sous des formes peu ou non marchandes. Le nombre de ménages dont un membre au moins pratique une activité agricole ou d'élevage ${ }^{12}$, estimé à 16000 par la direction de l'Agriculture et de la Forêt en 1997, atteint près de 21000 en 2003 (Huet, op. cit.). L'agriculture concerne ainsi près des deux tiers des ménages de l'île ${ }^{13}$, et occupe 20250 ha (Huet, op. cit.). Malgré sa croissance démographique exceptionnelle, Mayotte est par ailleurs toujours autosuffisante en bananes et tubercules, composantes végétales principales (avec le riz) du régime alimentaire. Les paysages de l'île témoignent d'une colonisation agricole sur des pentes de plus en plus fortes.

Le développement économique et la tertiarisation de l'économie mahoraise ne se font pas aux dépens de l'activité agricole mais en complémentarité avec elle. Celle-ci se maintient quels que soient les parcours professionnels des individus, soulignant la logique des Mahorais de complexification de leur système d'activités plutôt que de spécialisation (Sourisseau, 2003). L'activité agricole concerne tous les niveaux de revenus et toutes les catégories socio-économiques (Losch et Sourisseau, op. cit.) Le maintien de l'activité agricole ne se limite pas à des agriculteurs âgés persistant dans leur activité dans une logique de résistance. Un chef d'exploitation sur deux a moins de 40 ans (Huet, op. cit.) et la majorité des jeunes

12. En l'absence de statut pour les agriculteurs et du fait des spécificités de l'organisation sociale en milieu rural, cette définition du ménage agricole, proposée par la direction de l'Agriculture et de la Forêt, nous paraît pertinente.

13. Ces chiffres incluent les étrangers. Selon Huet (op. cit.) près d'un tiers des chefs d'exploitations agricoles est d'origine comorienne, ce qui est conforme au poids estimé des étrangers dans la population. L'enquête ne permet pas de savoir si ces exploitants sont en situation irrégulière, mais le fait que $76 \%$ d'entre eux accèdent à la terre via un bail oral ou une mise à disposition gracieuse suggère qu'il s'agit essentiellement de clandestins. ménages démarre une activité agricole dès le mariage ou à la naissance d'un premier enfant (Sourisseau, op. cit.).

Allant à l'encontre des messages des services techniques, la grande majorité des ménages agricoles met en œuvre des systèmes de production complexes, construits autour d'un assemblage d'une dizaine d'espèces végétales sur des parcelles le plus souvent inférieures à 1 ha $^{14}$, éventuellement combiné à une production animale à petite échelle. Ces systèmes ont traversé l'histoire agricole de l'île en conservant leur structure. Le bananier et le manioc en sont les productions dominantes, concernant $97 \%$ des ménages agricoles et couvrant plus de $80 \%$ des surfaces. Elles sont conduites en association avec des fruitiers plantés ou sauvages (manguiers et jacquiers notamment) et d'autres composantes importantes de la cuisine locale : ambrevades ${ }^{15}$, noix de coco, courges, piments, aubergines, ananas, etc. La préparation, l'agencement et l'entretien des parcelles sont essentiellement manuels et se gèrent à la micro-parcelle, en tenant compte du relief, souvent accidenté, et des propriétés du sol. La diversité des combinaisons des espèces végétales et des densités culturales est ainsi remarquable. L'élevage bovin, caprin et/ou ovin concerne un tiers des ménages agricoles; les troupeaux comptent généralement moins de 10 têtes, $80 \%$ des animaux divaguent ou sont attachés individuellement (Huet, op. cit.). Pour les bovins notamment, les pâturages spécialisés sont rares et la pratique de l'attache au piquet suppose une présence quotidienne ${ }^{16}$.

14. Si les surfaces moyennes par ménage sont de 1 ha (Huet, op. cit.), celles-ci sont le plus souvent morcelées.

15. Légume se consommant comme des petits pois, issu d'un arbuste de $2 \mathrm{~m}$ de haut dont les feuilles possèdent des vertus médicinales et constituent un bon fourrage. Elle est aussi appelé pois d'Angole ou ambrevade.

16. Pour des raisons de disponibilités foncières et du fait de la place spécifique des animaux d'élevage dans la société mahoraise, on ne fait pas travailler les bovins et il existe peu de pratiques de transfert de fertilité (pratique présente à Anjouan). 
Le ménage, le couple et ses enfants non mariés, unité de résidence articulée autour de la femme propriétaire de la maison ${ }^{17}$, constitue schématiquement la base des systèmes de production agricoles. Chaque époux dispose généralement d'une à trois parcelles de cultures (shamba) - sur les propriétés familiales (mundra) de leur père et/ou de leur mère - et peut décider de ne mettre en culture qu'une partie de cellesci. Le ménage dispose ainsi d'un espace de culture morcelé ; certaines parcelles se trouvent à proximité des lieux de résidence, d'autres à plus d'une heure de marche. Le transport, effectué à pied, en voiture ou en taxi, est à la fois consommateur de temps et très coûteux. Il représente une contrainte majeure pour le développement des stratégies marchandes (Casalegno, 2002).

Les performances technico-économiques de ces systèmes complexes et morcelés sont difficiles à mesurer avec les indicateurs usuels de performance. Les coûts de production concernent essentiellement le transport en voiture personnelle ou en taxi et le travail, dont le marché est perturbé par la clandestinité et par le niveau élevé des salaires légaux imposés par l'alignement sur la République. Les agriculteurs n'utilisent quasiment pas d'intrants et le matériel agricole est rudimentaire ${ }^{18}$. Le rendement à l'hectare n'est par ailleurs pas un critère opérationnel, du fait d'une gestion technique manuelle de la plante sur des surfaces très réduites; c'est au nombre de pieds que

17. Si elle est soumise à l'homme par le droit musulman, la femme occupe une position centrale du fait de l'application du principe de matri-localité. 18. Les dépenses en intrants sont quasiment nulles. Utilisant les rejets pour des bananiers ou le bouturage pour de nombreuses autres cultures, les ménages ont rarement recours à l'achat de pieds ou de graines. De plus, seuls $5 \%$ d'entre eux utilisent de l'engrais et $7 \%$ des pesticides. Par ailleurs, environ $90 \%$ des ménages n'utilisent que le shombo (machette) pour réaliser les travaux agricoles (Huet, op. cit.) se mesurent les quantités ${ }^{19}$. Enfin, comme nous l'avons vu, une très faible part des productions est vendue et la valorisation des autres utilisations passe rarement par une référence au marché. Dans ces conditions les systèmes de prix sont peu opérants. La stabilité et la permanence des systèmes que nous venons de décrire renvoient plutôt à leur capacité à satisfaire les fonctions assignées prioritairement à l'agriculture, autres que la production pour le marché.

Les principales productions végétales sont d'abord destinées à l'alimentation des ménages, les quantités plantées étant légèrement supérieures aux besoins du groupe familial. Si des produits nouveaux sont apparus dans l'alimentation via le développement du marché (viande surgelée, boissons sucrées, pâtes, etc.), la consommation de produits locaux a été peu modifiée ${ }^{20}$.

Pratiquer une activité agricole, c'est également disposer de produits alimentaires et symboliques qui offrent la possibilité de participer aux réseaux familiaux ou villageois d'échanges et d'affinité (Barthès, 2003). Ces réseaux fonctionnent selon le principe de réciprocité, socle de la culture comorienne (Blanchy-Daurel, 1990). S'y inscrire en fournissant une aide matérielle ou financière aux autres participants permet de développer des relations de proximité et d'entretenir son appartenance au groupe familial, à sa classe d'âge, à sa confrérie religieuse et, plus largement, à la communauté villageoise. Y participer active également pour un individu le droit de recevoir une aide en retour. Ceci n'a rien d'anecdotique. Tenter de se soustraire aux obligations de redistribution peut entraîner jusqu'à

19. Par suite, les éléments d'analyse de la SAU, mobilisés dans les principaux documents de présentation de l'agriculture mahoraise, doivent aussi être relativisés.

20. Seul le riz n'est quasiment plus produit sur l'île, essentiellement du fait de trop importants temps de travaux (récolte) et de l'introduction de subventions à l'importation doublées d'aides à l'implantation locale d'une usine de décorticage. 
l'exclusion de certains réseaux alors que les honorer garantit des liens sociaux et économiques indispensables. Les transferts de produits agricoles sont réalisés au quotidien et, de façon plus importante, lors des nombreuses manifestations à caractère religieux et coutumier qui rythment la vie mahoraise $^{21}$. Dans un contexte d'écart croissant des niveaux de vie entre les ménages salariés et ceux n'ayant pas de revenus sécurisés, les fonctions de redistribution assurées par l'activité agricole à travers le transfert de produits sont d'autant plus importantes.

Plus largement, cultiver garantit un ancrage dans son espace familial et villageois. L'activité agricole assure, au-delà d'une fonction identitaire ancienne $\mathrm{e}^{22}$, un maintien $\mathrm{du}$ patrimoine foncier familial. Cette fonction patrimoniale se trouve renforcée, dans une logique défensive, par la régularisation foncière en cours. L'activité agricole permet en effet de garantir l'activation des droits coutumiers de propriété et d'éviter qu'un tiers ne s'approprie la parcelle en la cultivant et en engageant des démarches de régularisation auprès des services fonciers.

Finalement les ménages agricoles vivant exclusivement de l'agriculture sont rares et les producteurs qui vendent une large part de leur production ont très majoritairement d'autres sources de revenus. La fonction de production de biens marchands n'en est pas pour autant négligeable. Elle offre en effet une solution de repli en cas de difficultés et des compléments de revenu appréciables pour les ménages les plus démunis. L'élevage bovin s'inscrit dans une logique marchande, en tant que lieu privilégié d'utilisation de l'épargne des ménages. Malgré le développement des institutions bancaires, les animaux sont toujours perçus comme des

21. Circoncisions, fiançailles, mariages, enterrements, célébrations en l'honneur des esprits, etc. 22. Seuls les individus qui ont des terres et qui sont nés au village ont le statut de «mudjeni » (natif du village). Grâce à ce statut, ils disposent d'un pouvoir de parole pour intervenir dans les affaires villageoises (Blanchy-Daurel, op. cit.). immobilisations de capital facilement démobilisables. Rarement investies dans l'appareil productif, les ventes contribuent plutôt aux financements des nombreuses et incontournables cérémonies et obligations sociales.

S'intéresser au caractère multifonctionnel de l'agriculture ${ }^{23}$ permet de mettre en lumière les spécificités de l'activité et sa distance par rapport à un modèle agricole métropolitain de fait exogène (Sourisseau et al, 2004a). La stabilité des fonctions non marchandes témoigne non seulement d'une résistance des ménages ruraux aux incitations des institutions républicaines, mais aussi d'un maintien des objectifs des ménages déterminés en grande partie par les institutions locales (la famille, les classes d'âges, les groupes de chants et de danses religieuses, le village). Il convient, en restant dans le cadre d'analyse de l'influence croisée de ces deux catégories d'institutions, d'étudier les modes d'organisation permettant cette relative stabilité.

\section{La réorganisation de l'activité agricole}

Selon les principes de l'organisation économique coutumière, les décisions techniques d'utilisation des moyens de production relèvent du ménage tandis que l'allocation des moyens et la valorisation des produits sont soumises à un contrôle collectif de la famille, de la classe d'âge des individus ou d'autres groupes différemment constitué généralement à l'échelle du village (Rafaël, 2003). Ce cadre de référence s'applique aux activités agricoles, et notamment à ses formes peu marchandes que nous venons de décrire. Il reste aujourd'hui effectif mais a

23. D'autres fonctions peuvent également être citées : maintien des populations en zone rurale dans un contexte de forte pression démographique, entretien des paysages et sentiers agricoles, lutte contre l'érosion et l'envasement du lagon, gestion des ressources naturelles, etc. 
dû s'adapter, notamment face aux réajustements de la gestion du foncier et du travail agricole induits par les modifications de l'environnement institutionnel et les mutations économiques et sociales

\section{Adaptation et résistance des modes de gestion du foncier}

Jusqu'à aujourd'hui, le foncier se gère essentiellement selon les principes du droit local, alliant droits coutumier et musulman (Barthès, op. cit.). Une terre est considérée comme appartenant à une famille si ses ancêtres possédaient autrefois cette terre ou si une personne de la famille l'a mise en valeur. Au sein du groupe familial, le foncier est géré par un ayant droit principal, désigné comme chef des terres. Celui-ci seul possède le droit de vendre la terre et d'en répartir les droits d'usages entre les ayants droit ${ }^{24}$.

Dans le cadre de la mise aux normes institutionnelle, un programme de régularisation foncière en cours vise à enregistrer et immatriculer l'ensemble du foncier de Mayotte selon le droit commun. Il s'agit schématiquement de substituer définitivement l'appropriation coutumière régulée par des logiques familiales et villageoises par la propriété privée régulée par le marché et les services publics. L'administration recherche notamment par cette mesure à créer un marché foncier, à clarifier et à rationaliser les principes d'allocation de la terre (Bonnelle, 1998). Un des objectifs affichés est de sécuriser la propriété, en particulier pour les agriculteurs, pour qui la gestion familiale représenterait un frein à l'investissement (Barthès, op. cit.).

Pour rendre effective la régularisation, d'importants moyens sont déployés. Mandatés par le Conseil général, des agents du Centre national d'aménagement des structures des exploitations agricoles (Cnasea) enregistrent commune par

24. Le respect du partage est contrôlé, entre autres, par les représentants villageois de la justice musulmane et les notables fondateurs du village. commune toutes les parcelles non encore titrées. Ces dernières font ensuite l'objet d'un bornage soumis à la population par voie d'affichage, charge ensuite au particulier, si sa propriété n'est pas contestée, de régulariser sa situation auprès des services fiscaux. Cette démarche, au demeurant payante jusqu'en 2005, est indispensable pour valider la régularisation et permettre au propriétaire du terrain de jouir pleinement de ses droits.

En ajoutant un nouveau mode d'appropriation, la régularisation génère de nouveaux risques de revendication des terrains par des tiers. Par ailleurs, les terres non revendiquées et titrées seront à terme reversées dans le domaine public, entraînant une dépossession de fait de leurs anciens occupants. Le passage par le processus d'immatriculation apparaît ainsi incontournable. Pour autant la démarche, coûteuse et fastidieuse, n'est entreprise que lorsque la menace de spoliation est jugée crédible. Si de nombreuses familles s'y engagent, seules quelques-unes vont au bout du processus en payant effectivement l'enregistrement aux services des domaines (Barthès, op. cit.). Le fait de poser les bornes apparaît comme un signe suffisant pour protéger sa propriété tout en évitant les coûts légaux. Les groupes familiaux affichent une adhésion de façade mais cherchent à maintenir des modes de gestion mieux maîtrisés.

Pour se prémunir d'un risque de contestation de leurs droits fonciers, nous avons vu que les familles s'appuient aussi sur le droit local en mettant leurs parcelles en culture. La régularisation a ainsi entraîné une augmentation des surfaces cultivées plutôt qu'une d'intensification en capital des systèmes de production. Pour mettre en œuvre cette stratégie défensive, les ménages engagent plus de main-d'œuvre, exclusivement en situation irrégulière, ou cèdent temporairement leurs parcelles aux migrants clandestins qui, du fait de leur situation illégale, ne sont pas en mesure d'engager des démarches de régu- 
larisation. Ainsi la traduction locale des incitations visant à rapprocher Mayotte du cadre légal républicain aboutit ici à l'émergence de nouvelles formes de régulations distantes voire en contradiction avec les objectifs initiaux.

Le processus de régularisation n'a pas changé radicalement l'allocation du patrimoine foncier, même s'il peut être à l'origine de pratiques opportunistes et de conflits fonciers intra ou interfamiliaux. Lorsque les parcelles sont bornées, elles délimitent des ensembles familiaux et rarement les lopins individuels. Ainsi le chef des terres garde son autorité sur les ayants droit en leur refusant de fait le pouvoir de gérer pleinement une parcelle et l'accès effectif au marché du foncier. Même ceux qui parviennent à acheter dans le respect du droit commun, en dehors du patrimoine familial, ne parviennent pas à instaurer une propriété privée et individuelle complète. S'ils gagnent en autonomie de gestion par rapport à leur groupe familial, ils n'échappent pas à leurs obligations envers leur fratrie, et plus encore envers leurs enfants. Dans le même temps, le contrôle collectif garantit une certaine flexibilité de la gestion familiale et solidaire du foncier, l'allocation des terres pouvant aisément s'adapter aux besoins et aux capacités des membres de la famille ${ }^{25}$.

En jouant du pluralisme institutionnel et légal introduit par la régularisation, les acteurs individuels et collectifs parviennent pour l'heure à contrôler la spéculation et la concentration foncières. Alors que l'on pouvait s'attendre à des mouvements violents sur un marché émergeant dans un contexte de raretée ${ }^{26}$, la stabilité des institutions locales garantit encore un accès à la terre agricole pour le plus grand nombre.

25. Par exemple, une veuve se verra attribuer une parcelle proche de chez elle, parfois au détriment d'un autre membre ; un homme dépendant de l'agriculture pourra disposer de façon temporaire d'une plus grande parcelle pour faire de l'élevage, etc. 26. Ces phénomènes sont du reste à l'œuvre pour le foncier urbain constructible.
Ainsi les ventes de terres agricoles clairement appropriées par des familles sont rares et le marché de la location est peu actif ${ }^{27}$. À l'inverse, le prêt reste une pratique courante et s'établit, selon un principe de réciprocité, entre parents ou entre résidents d'un même village ; le bénéficiaire pouvant être Mahorais ou Comorien en situation irrégulière (Burnod, 2007).

\section{La réorganisation de la gestion du travail}

Le couple et ses enfants se répartissent les tâches agricoles selon leur pénibilité et l'éloignement de la parcelle : les enfants et les femmes gèrent préférentiellement l'entretien des cultures et interviennent plutôt sur les parcelles proches, les hommes le défrichage et la plantation sur les parcelles éloignées. Le développement de l'emploi salarié et l'augmentation des revenus modifient les conditions d'allocation du temps et du travail de chaque actif. En effet, de nouvelles opportunités de revenus hors agriculture sont offertes, tandis que la population comorienne immigrée propose du travail à bas prix.

Plus de $20 \%$ des ménages agricoles, en majorité des ménages dont le chef d'exploitation est mahorais, délègueraient une partie ou l'ensemble du travail agricole. Il s'agit pour eux de moduler le temps passé dans les champs pour se consacrer à d'autres activités plus rémunératrices, moins physiques ou plus adaptées à leurs besoins. Ainsi, de nombreux contrats de travail illégaux sont établis avec des migrants clandestins. De façon schématique, seuls les ménages dont un des membres dispose d'un emploi à plein temps délèguent à un tâcheron les travaux tels que le sarclage et le défrichage des parcelles et la plantation (bananier et manioc). Dans le cadre de ces

27. Le niveau des loyers, notamment dans le cas de production pour l'autoconsommation, s'apparente plus souvent à une contribution symbolique qu'à un véritable prix de marché (Burnod et Colin, 2005a). 
contrats à la tâche, la rémunération journalière implicite du travail est comprise entre 6 et 10 euros $(€)$. Les ménages les plus aisés délèguent à un ouvrier rémunéré mensuellement la conduite de l'élevage bovin (lorsqu'ils sont propriétaires de plus de 5 à 6 têtes) et/ou l'entretien quotidien des cultures (lorsqu'ils disposent d'une parcelle de plus d'un demi-hectare dans des zones agricoles enclavées). Les ouvriers mensualisés perçoivent 100 à $150 €$ par mois, soit moins d'un quart du Smig mahorais. Ces contrats de travail impliqueraient ainsi plus de 10000 Comoriens en situation irrégulière ${ }^{28}$. Le recours à ces arrangements illégaux, fréquent dans les secteurs agricole et du BTP, est renforcé par la difficulté de payer un ouvrier au taux légal. Celui-ci, fixé arbitrairement dans une logique d'alignement mécanique sur la métropole étant déconnecté de la productivité effective de l'économie locale. Par contrecoup, les pratiques collectives d'entraide en travail (musada), fondées sur la réciprocité et autrefois courantes, ont tendance à disparaître.

Les dynamiques décrites illustrent le développement de relations à caractère marchand. Pour autant les contrats de travail ne sont pas systématiquement réductibles à un simple échange marchand (Burnod op. cit.). Parfois, la relation entre employeursaccueillants mahorais et employés-accueillis se compose d'un faisceau de transactions marchandes et non-marchandes. Les employés et leur ménage bénéficient d'un logement, d'une aide matérielle ou financière ponctuelle, du droit de cultiver leur propre lopin ; ils peuvent aussi être invités à partager les repas chez leurs employeurs. Ces échanges, régulièrement étudiés dans le cadre de relations de patronage (Platteau, 1995), sont analysés dans la littérature économique comme des contrats liés incitant l'employé à bien travailler (Bardhan, 1980 ; Otsuka et al, 1992). Dans la situation maho-

28. Du fait de l'illégalité de cette pratique, il est possible que ces chiffres soient sous-estimés. raise, ces transactions peuvent s'inscrire dans une logique de réciprocité qui dépasse largement le rapport de travail et sa contrepartie. L'accès à la terre ou à un logement n'est pas lié à la prestation de travail et perdure une fois l'échange de travail accompli ${ }^{29}$. En contrepartie des services dont les migrants et parfois leur ménage bénéficient, ils proposent à leur employeuraccueillant et à sa famille d'effectuer divers travaux (bricolage, garde des enfants, tâches ménagères) et participent à l'organisation de manifestations religieuses, comme il est attendu dans une relation entre proches. Ainsi, le bon déroulement de la transaction marchande de travail ne s'explique pas uniquement par des effets d'incitation mais aussi par la volonté des contractants d'être loyaux avec leur partenaire ${ }^{30}$.

La dimension identitaire est ainsi très prégnante, quand bien même la menace d'un recours à la loi pour faire respecter les termes du contrat lui est généralement associée (Burnod et Colin, 2005b). De la même façon que pour la gestion foncière, on assiste à une hybridation des pratiques contractuelles - entre réciprocité et marché - liée au pluralisme institutionnel introduit par les logiques de l'intégration.

\section{Les adaptations du contrôle social de l'activité agricole}

Les décisions concernant l'utilisation des produits agricoles ne relèvent pas exclusivement du couple gérant l'unité de production. L'engagement de chaque individu dans un jeu d'échanges réciproques

29. De plus, l'employeur et sa famille peuvent accueillir temporairement un enfant du migrant, donner des vêtements à ses enfants et participer au financement de cérémonies religieuses.

30. L'analyse détaillée de certains cas montre par ailleurs que lorsque les partenaires viennent à se considérer comme frères, ou père et fils, et que le migrant est présenté comme tel dans la famille de son partenaire mahorais, la relation de travail peut perdre son caractère marchand (Burnod et Colin, 2005a). 
au sein de ses lignages et village se traduit par des obligations de dons plus ou moins prévisibles. Si l'inscription dans ces réseaux procure des avantages en activant des liens solidaires et des droits pour l'individu mis à contribution, elle peut s'avérer contraignante pour mettre en œuvre des stratégies individuelles de commercialisation, voire d'investissement.

D'une manière générale l'absence de maîtrise individuelle liée au contrôle collectif des produits du travail ne favorise pas l'expression d'une logique de production strictement marchande. La volonté des ménages vis-à-vis de leur activité agricole peut être d'augmenter les rendements mais les objectifs sont rarement ceux d'une meilleure profitabilité. Dans ce contexte, les projets d'accroissement de la productivité par un effort d'investissement en capital prônés par les services techniques trouvent peu d'écho. Se référant au modèle agricole français, jugé moderne et souhaitable par rapport aux pratiques locales, les différents programmes d'aide à l'installation et à la modernisation qui se sont succédés ont reçu très peu de demandes ${ }^{31}$. Plus encore, les individus en ayant bénéficié peinent à mettre en place une réelle dynamique marchande et conservent de toute façon d'autres sources de revenus.

Pour les producteurs, la spécialisation de leur système de production et la limitation des prélèvements familiaux représentent un risque social, venant s'ajouter aux difficultés de rentabiliser et de pérenniser une exploitation agricole sur un mode

31. Par exemple, entre 1997 et 2001 , seules 180 décisions d'aide à l'installation ou à la modernisation ont été validées en Commission départementale d'orientation en agriculture (CDOA). La simplification des procédures a permis par la suite une augmentation des aides, mais dans des propositions très faibles par rapport aux 21000 ménages agricoles recensés. marchand $^{32}$. Par ailleurs, le développement d'un système spécialisé, engageant sur le long terme, ne semble pas correspondre aux objectifs des producteurs. Ceux-ci recherchent plutôt la souplesse de gestion et la réversibilité. Dans cette logique, les producteurs bénéficiant d'aides à la modernisation ou à l'installation finissent souvent par déléguer le travail à des clandestins et, par suite, n'acquièrent pas les compétences techniques. Ainsi, il n'y a pas malgré les incitations fournies par les services publics de changement conséquent des structures de production existantes. La mobilisation dans le champ de l'entreprise des institutions anciennes de contrôle collectif des pratiques individuelles explique pour partie ce manque de réponse ${ }^{33}$.

\section{La redéfinition de la place de l'agriculture dans les systèmes d'activités}

Bien plus que les pratiques agricoles et leurs performances intrinsèques, ce sont le poids et la place de l'agriculture au sein des systèmes d'activités développés par les ménages agricoles qui différencient ces derniers et permettent d'isoler leurs grandes orientations stratégiques (Losch et Sourisseau, op. cit. ; Barthès, op. cit.). Or, la composition et la dynamique de ces systèmes d'activités sont influencées par les changements institutionnels liés à l'intégration de Mayotte dans la République.

32. Mayotte est, comme toutes les petites économies insulaires, soumise aux contraintes structurelles d'un marché intérieur étroit et d'une compétitivité grevée par la faiblesse des économies d'échelle potentielles, une géographie difficile et l'éloignement des grandes routes commerciales.

33. Le maraîchage, seule production marchande qui se développe sur des modes susceptibles de rencontrer l'offre d'appui, ne peut être aidé du fait de la situation illégale de la majorité des producteurs. 
Sous l'effet de la rente administrative ${ }^{34}$, l'augmentation sensible des offres d'emplois relativement bien rémunérés pénalise très fortement les initiatives entrepreneuriales; et en particulier dans le secteur agricole. À l'inverse le maintien d'une activité agricole, dont nous avons vu l'importance, ne doit pas empêcher de saisir ces opportunités offertes sur le marché du travail. Il s'agit de mettre en œuvre des combinaisons d'activités économiques et sociales adaptables, dont la plasticité doit s'exprimer dans le temps court comme à l'échelle des cycles de vie des individus.

De façon générale les ménages mahorais privilégient la sécurité et la régularité des revenus, en accordant leur préférence au salariat, si possible dans la fonction publique $^{35}$. La pluriactivité est perçue comme un facteur de dispersion des risques économiques et, par suite, les stratégies de spécialisation dans une activité indépendante sont rares. Il leur est préféré un engagement sectoriel minimal et réversible, économe en temps et en capital, qui peut être réactivé dès que des besoins financiers particuliers apparaissent, et notamment les dépenses cérémonielles, prévisibles ou non. Car en plus de sa disponibilité pour sa communauté, tout Mahorais(e) doit assurer schématiquement son grand mariage, la circoncision de ses fils, les études de ses enfants, la construction de la maison de ses filles, la célébration de ses deux parents décédés (madziho) et son pèlerinage à la Mecque. Cette série d'obligations dans un contexte de contrôle collectif des moyens de production impose des anticipations qui influencent les trajectoires professionnelles.

34. Le secteur des administrations représentait plus de $40 \%$ du PIB local en 2001 (Frouté et Massuyau, op. cit.). Mayotte n'échappe donc pas au phénomène de rente administrative, caractéristique de la situation des DOM TOM.

35. Les niveaux de rémunération et leur progression bi-annuelle en vue de rattraper les taux métropolitains expliquent largement cette préférence.
L'âge d'entrée dans la vie active correspond à l'âge d'accès à une parcelle en propre, au moment du premier mariage (Sourisseau, op. cit.). Par la suite la permanence de la pratique de l'agriculture vivrière est remarquable. Sauf rares exceptions, elle n'est arrêtée qu'en fin de cycle de vie, du fait de l'âge et de l'incapacité physique. Cette permanence contraste avec l'instabilité qui prévaut sur la longue période à la mise en œuvre des autres activités.

L'artisanat et les prestations de services sont démarrés généralement à plus de trente ans. Ces activités sont entreprises de façon temporaire dans l'optique de satisfaire des besoins spécifiques : achat de meubles, éducation des enfants, organisation d'une cérémonie, etc. Une fois l'argent nécessaire récolté, l'activité productive peut être arrêtée, puis éventuellement reprise en cas de nouveau besoin ${ }^{36}$. Le salariat est aussi très fréquent dans les systèmes d'activités et sa place se renforce depuis la fin des années 1980. Alors qu'auparavant les contrats précaires de courte durée dominaient, on constate un allongement de la durée des contrats salariés, qui offrent du coup de réelles perspectives d'accumulation. On constate également que les contrats démarrent plus tôt que par le passé, ce qui élargit la gamme de choix des jeunes actifs.

La place de l'agriculture marchande dans les systèmes est elle aussi marquée par le tournant de la fin des années 1980. Outre la régression de l'ylang-ylang, de la vanille et du riz, c'est durant cette période charnière que se développent les transferts sociaux de l'État et qu'émerge un marché des produits alimentaires de base. Toutefois l'opportunité de vendre ne se traduit que

36. Les entreprises de commerce et d'artisanat sont nombreuses mais peu équipées. Dans une logique proche de celle rencontrée pour l'agriculture, elles emploient peu de personnels et ont une activité erratique. Les entrepreneurs privilégient la réversibilité et la souplesse de gestion du travail et des capitaux, au détriment des performances technicoéconomiques. 
rarement par une stratégie de spécialisation. Les ménages y ont recours par période. Sans une refonte des systèmes de production nécessitant des investissements conséquents, difficilement réversibles et donc hautement risqués, les revenus agricoles ne permettent pas d'enclencher ou d'entretenir un processus d'accumulation. L'activité se maintient donc en articulation avec d'autres.

L'élevage occupe toujours une place à part dans les cycles de vie, puisqu'il est peu développé pour ses fonctions alimentaires. Le lait, généralement laissé aux veaux, et la viande ne sont consommés qu'à l'occasion de célébrations. En revanche, l'achat d'un bovin correspond au même titre que l'immobilier à un choix prioritaire de placement pour bien des ménages. Une première stratégie consiste à devancer les grandes dépenses programmables et à affecter spécifiquement un atelier d'élevage à la réalisation d'un objectif donné. Chaque préparation d'une cérémonie donne lieu au démarrage d'un atelier et l'activité d'élevage est arrêtée une fois l'objectif atteint. À l'inverse, un individu peut choisir de maintenir en permanence quelques bovins, pour faire face à une éventuelle situation difficile. Cette seconde option apparaît plus tard dans les cycles de vie. D'une manière générale, l'investissement en temps dans l'agriculture est plus important en fin de vie, lorsque l'accès au salariat et aux autres activités se réduit.

La pluriactivité des individus qui composent les ménages agricoles apparaît donc comme une constante à toutes les étapes de leur cycle vie (Barthès, op . cit. ; Sourisseau et al, 2004b). Quoique prenant des formes diverses, les systèmes d'activités se construisent généralement autour d'une agriculture à base vivrière stable mais modulable vers des fonctions plus marchandes en fonction des besoins. Leur dynamique est par ailleurs jalonnée de grandes périodes qu'il est possible de schématiser grossièrement.

Les premières années d'activité, concomitantes avec l'accès au foncier sur la parcelle familiale, visent à stabiliser l'agriculture sur un plan technique et institutionnel. Puis, une ou plusieurs activités plus rémunératrices s'articulent à l'agriculture vivrière. Ces activités peuvent être ponctuelles (salariats précaires, petits boulots, vente de produits agricoles issus des parcelles initialement vivrières) ou plus stables (maraîchage spécialisé, CDI, artisanat stable). Durant cette seconde phase se concrétisent les mariages et les premières obligations envers les enfants, tandis que les individus entrent dans le jeu de dette inhérent à leur inscription dans leur communauté. Il leur faut donc produire pour sécuriser l'alimentation et pour donner. La délégation des travaux, financée par les revenus non agricoles, est parfois nécessaire pour maintenir un niveau de production couvrant ces besoins alimentaires et sociaux. Sinon, du fait des obligations scolaires des enfants et du temps consacrés aux autres activités, les travaux s'effectuent le weekend. La production agricole pour le marché relève soit d'une délégation plus importante du travail, soit de l'allocation du travail familial vers l'agriculture lorsque les autres opportunités de revenus sont inaccessibles. L'ajustement se fait par augmentation des surfaces cultivées - par récupération de parcelles temporairement cédées ou par des prêts - plutôt que par un changement des systèmes de culture. La préférence reste accordée au système multi-espèces traditionnel finalement peu exigeant en travail pour satisfaire les besoins de base et facilement extensible sans investissement.

Dans une troisième phase, les systèmes se complexifient encore, en même temps que certaines activités se structurent et que les possibilités d'accumulation s'accroissent ${ }^{37}$. Les transferts monétaires vers l'agriculture sont aussi davantage sécurisés, ce qui facilite l'orientation des systèmes techniques.

37. En particulier pour les salariés qui ont alors de l'ancienneté. 
Dans le même temps les obligations des individus envers leur communauté se renforcent. Les ménages cherchent alors à diversifier leur production agricole et à développer des élevages bovins ou caprins, tant pour répondre à ces obligations que pour consolider les systèmes d'activités. Les mécanismes de délégation du travail et d'agrandissement foncier sont alors mis en œuvre. Selon la régularité et l'importance de la principale source de revenu du ménage et donc des transferts, cette diversification peut signifier une marchandisation de l'agriculture. Elle s'accompagne alors d'une intensification en capital de l'activité, de l'introduction d'innovations, de demande d'aides publiques et du développement du maraîchage ou de vergers. Mais le plus souvent elle passe par une complexification du système de production initial, avec l'introduction de nouvelles espèces et l'ouverture de nouvelles parcelles plus spécialisées mais non destinées à la vente. On note aussi le développement d'ateliers ponctuels d'élevage.

La fin des cycles d'activités est réservée à l'entretien et au renforcement de la reconnaissance sociale (pèlerinage, participation active dans la vie cérémonielle des villages, etc.), qui sont facilités par l'agriculture et ses fonctions identitaires. On note parfois un investissement dans l'agriculture et l'élevage marchands ; certains retraités ayant construit progressivement leur exploitation agricole autour du vivrier visent une augmentation des performances agricoles une fois leur activité principale arrêtée. Outre une intensification en travail, la recherche de gains de productivité peut alors passer par l'adoption d'itinéraires techniques plus productifs (recours à davantage d'intrants, réduction du nombre d'espèces végétales par parcelle, etc.). Mais le plus souvent les systèmes de culture se recentrent sur le vivrier, le temps disponible étant utilisé pour améliorer l'entretien des plantes et limiter la délégation de travail.
La permanence de l'agriculture tout au long des trajectoires est rendue possible par la souplesse intrinsèque des systèmes de culture, renforcée par les ajustements des gestions du foncier et du travail que nous avons analysés plus haut. Malgré son faible poids dans la structure des revenus des ménages, l'activité agricole joue donc un rôle de pivot au sein des systèmes d'activités. Son inscription tout à la fois dans des relations marchandes et nonmarchandes est un élément déterminant des orientations stratégiques des trajectoires. Ainsi sa connexion permanente avec les besoins de la famille et du groupe villageois d'appartenance contribue tout au long des cycles d'activité à la densification des réseaux de relations interpersonnelles. Par la suite, ces réseaux facilitent le raisonnement stratégique en élargissant l'éventail des choix d'activités.

\section{Conclusion}

À Mayotte, le changement institutionnel a ouvert largement le champ des opportunités offert aux ménages agricoles, mais n'a pas modifié radicalement leurs pratiques et leurs logiques. S'il s'est accompagné de nouvelles contraintes (procédures administratives, Code du travail), il a surtout généré de nouvelles incitations (subventions, appui technique et organisationnel, opportunités de revenu hors agriculture). Contrairement à ce que prévoyaient les services de l'État et les politiques locaux, ces incitations n'ont pas généré de signaux suffisamment lisibles ou pertinents pour amorcer la mutation du monde rural local vers un modèle agricole marchand. On attendait une concentration de la population agricole au sein d'exploitations de plus grande taille et mieux équipées, une spécialisation des systèmes de production et un développement de logiques commerciales. On observe au contraire la permanence d'une agriculture ayant pour fonctions principales de nourrir les nombreux ménages la pratiquant et d'entretenir les liens communautaires. Cette permanence s'explique par l'adaptabilité des techniques culturales traditionnelles - qui 
perdurent dans l'histoire - mais aussi par la réorganisation récente de la gestion du foncier et du travail agricole. La souplesse de gestion ainsi acquise autorise d'articuler l'agriculture à d'autres activités au sein de systèmes complexes.

Les réactions au changement institutionnel en cours ne se font pas comme cela avait été envisagé dans une logique de substitution. Elles donnent lieu au contraire à un processus d'empilement, où les règles, normes ou droits d'origine exogène viennent s'additionner aux institutions locales. Si les institutions dites formelles peuvent être changées rapidement, il apparaît que l'évolution des institutions dites informelles est en revanche beaucoup plus lente (North, op. cit.). Cristallisant le savoir, les valeurs et l'expérience des Mahorais depuis des générations, les institutions locales se maintiennent ou s'adaptent progressivement. L'irrigation des réseaux selon les principes de réciprocité, le rôle des autorités parentales dans la gestion du foncier, les obligations intergénérationnelles orientant les activités d'élevage sont autant d'institutions qui se reproduisent malgré le changement d'environnement. D'autres institutions se modulent : les journées d'entraide villageoises sont de moins en moins fréquentes et le recours à de la maind'œuvre salariée se développe. D'autres enfin se transposent, comme les principes de réciprocité qui s'immiscent au sein de relations de travail initialement marchandes. Ces changements sont le fruit des réponses des ménages à différents ordres normatifs et à l'évolution de leurs préférences. S'appuyant sur les différents niveaux institutionnels se présentant à eux, ils réagissent selon des modes hybrides. On retrouve ainsi un constat, déjà fait par ailleurs, à savoir que l'impact du changement institutionnel sur les pratiques des acteurs ne peut être compris sans prendre en compte l'influence, complémentaire ou antagonique, des institutions dans leur ensemble, formelles et informelles (North, op. cit. ; Hodgson, 1998 ; Schmid, op. cit.).

Le choix d'intégrer la République n'est pas pour les décideurs publics un choix de compromis mais d'acceptation totale de ces institutions. Le scénario d'imposition - voire de transposition - choisi à Mayotte et mis en œuvre sur des pas de temps très courts, affiche pour l'instant des résultats mitigés et génère même des effets allant à l'encontre des objectifs fixés. Ainsi le travail clandestin et des transactions foncières informelles se développent, l'instauration d'un cadastre foncier rencontre de nombreuses difficultés, la mise en culture de parcelles sur des zones à fort risque d'érosion et le défrichage de zones forestières en vue de marquer sa propriété sont fréquentes, etc. Dans le même temps, ces résultats peuvent être considérés comme positifs dans la mesure où la permanence des fonctions non marchandes de l'activité agricole permet de maîtriser certaines tensions et ruptures émergentes.

\section{RÉFÉRENCES BIBLIOGRAPHIQUES}

Bardhan P. (1980). Interlocking Factor Markets and Agrarian Development: A Review of Issues. Oxford Economic Papers, 32, p. 82-98.

Barthès C. (2003). L'État et le monde rural à Mayotte. Cirad-Karthala, collection Économie et développement, 221 p.

Blanchy-Daurel S. (1990). La vie quotidienne à Mayotte. Paris, L'Harmattan, collection « Repères pour Madagascar et l'Océan Indien ».

Bonnelle F. (1998). Réflexions sur l'avenir institutionnel de Mayotte. Rapport au secrétaire d'État à l'outre-mer. Paris, La documentation française.

Burnod P. (2007). L'enchâssement institutionnel des modes d'accès à la terre et au travail dans un contexte de migra- 
tion: le cas du secteur agricole à Mayotte. Thèse en économie du développement agricole, agroalimentaire et rural, Supagro, Montpellier.

Burnod P., Colin J.-Ph. (2005a). Changement institutionnel et immigration : recomposition et enchâssement social du marché des contrats agraires à Mayotte. Communication au colloque de la SFER, «Les institutions du développement durable des agricultures $d u$ Sud», Montpellier, 7-9 novembre.

Burnod P., Colin J.-Ph. (2005b). Napoléon Code versus Local Customs? Institutional Pluralism and the Enforcement of Agrarian Contracts in Mayotte. Annual conference of the International Society for New Institutional Economics, Barcelona, Spain.

Casalegno P. (2002). Étude de l'organisation et du fonctionnement des filières de commercialisation des produits agricoles vivriers traditionnels sur l'île de Mayotte. Rennes, Ensar, Cirad Mayotte, Sodel, $104 \mathrm{p}$.

Hodgson G. (1998). The Approach of Institutional Economics. Journal of Economic Literature, 36(1), p. 166-192.

Huet S. (2004). Enquête Statistique sur l'Agriculture et la Pêche en 2003. Premiers résultats. Direction de l'Agriculture et de la Forêt, Insee, Mamoudzou, Agreste Mayotte, $\mathrm{n}^{\circ} 1,4 \mathrm{p}$.

Losch B., Sourisseau J.-M. (Coord.) (2002). Quels place et rôles pour l'agriculture à Mayotte ? Bilan diagnostic du développement local. Mamoudzou, Montpellier, Cirad Mayotte, Sodel, $234 \mathrm{p}$.

North D. (1990). Institutions, Institutional Change and Economic Performance.
Cambrigde: Cambridge University Press.

Otsuka K., Chuma H., Hayami Y. (1992). Land and Labour Contracts in Agrarian Economies: Theories and Facts. Journal of Economics Literature, volume 30, p. 1965-2018.

Platteau J.-Ph. (1995). A Framework for the Analysis of Evolving Patron Client Ties in Agrarian Economies. World Development, vol. 23, $\mathrm{n}^{\circ}$ 5, p. 767-786. Rafaël B. (2003). Les dynamiques collectives sur le territoire du GIP Nord. Montpellier, Master-Valor Cnearc, Cirad Mayotte, Sodel, 176 p. + annexes. Schmid A. (2004). Conflict and Cooperation: Institutional and Behavioral Economics, Blackwell.

Sourisseau J.-M., Losch B., Mercoiret M.R. (2004a). Les dimensions économiques et sociales du développement durable à Mayotte : pour une approche territoriale. In Mayotte dans la République, actes du colloque de Mamoudzou, 14, 15 et 16 septembre 2002, CRJ, Université de La Réunion, Montchrestien, Collection Grands Colloques, p. 617-635.

Sourisseau J.-M., Mouridi Bina, Burnod P., Bonnal Ph., Losch B. (2004b). Le monde agricole mahorais, une lecture par l'articulation de relations marchandes et non marchandes. Dispositif conjoint InraCemagref-Cirad de recherche et d'expertise sur la multifonctionnalité de l'agriculture et des espaces ruraux, Mamoudzou, Montpellier, Document de travail Cirad Tera, $43 \mathrm{p}$.

Sourisseau J.-M. (2003). Bilan des activités du programme Sodel. Comité tripartite État CDM Cirad 2003. Mamoudzou, Cirad Mayotte, Sodel, 68 p. 